\title{
La analgesia peridural en fases tempranas del trabajo de parto no aumenta el riesgo de cesárea
}

\section{Objetivo}

Comparar la tasa de cesárea cuando se elige analgesia peridural versus analgesia sistémica, en pacientes nulíparas que se encuentren en fases tempranas de trabajo de parto.

\section{Diseño}

Ensayo clínico controlado aleatorizado no ciego.

Lugar

Centros de obstetricia, EEUU.

\section{Pacientes}

Se incluyeron mujeres sanas nulíparas, con embarazo único de término, en trabajo de parto espontáneo o con ruptura espontánea de membranas. Se excluyeron aquellas pacientes con presentación no cefálica, parto inducido, contraindicación para uso de opioides y dilatación cervical igual o mayor a $4 \mathrm{~cm}$.

El tipo de analgesia se aleatorizó a través de sobres opacos cerrados. La secuencia de asignación fue generada por computadora.

\section{Intervención}

En el grupo intervención, a la primera solicitud de analgesia, las pacientes con dilatación cervical menor a $4 \mathrm{~cm}$ recibían una dosis espinal de fentanilo. Simultáneamente, se colocaba un catéter peridural y en el segundo pedido de analgesia (si persistía la dilatación cervical menor a 4), se administraba una dosis de bupivacaina y fentanilo y luego un protocolo de analgesia regulado por la paciente a dosis prefijadas.

En el grupo control, al primer pedido de analgesia las pacientes con dilatación menor a $4 \mathrm{~cm}$ recibían una dosis de hidromorfona parenteral, repitiéndose la misma dosis al segundo pedido, siempre y cuando la dilatación continuase siendo menor a $4 \mathrm{~cm}$.

En ambos grupos ya fuera al tercer pedido de analgesia, o cuando se constatara una dilatación cervical mayor a $4 \mathrm{~cm}$ se administraba analgesia peridural según esquema tradicional (combinación de bupivacaina y fentanilo, con protocolo regulado por la paciente). El diseño no permitió cegar a los participantes ni a los médicos.

\section{Medición de resultados principales}

Punto final primario: Forma de finalización del embarazo (parto vaginal o cesárea).

Puntos finales secundarios: La causa de indicación de cesárea, modo de parto vaginal (instrumental o no), calidad de la analgesia, uso de ocitocina, duración del trabajo de parto, sufrimiento fetal, vitalidad neonatal.

\section{Resultados principales}

Se incluyeron 750 pacientes. Los grupos fueron homogéneos excepto por un mayor porcentaje de roturas de membranas y una dilatación cervical promedio menor en el grupo control. El análisis fue por intención de tratar.

No se encontraron diferencias significativas en la tasa de cesárea entre ambos grupos (diferencia porcentual de $-2.9 \%$, IC -9 a 3; $\mathrm{p}=0.31$ ). Tampoco se encontraron diferencias en la tasa de instrumentación vaginal (diferencia 3.6\%, IC -2.9 a 10; $p=0.13$ ), tasa de cesárea entre diferentes profesionales, valores promedio de temperatura basal, ni en el pronostico neonatal. (Ver tabla).

La duración de la primera fase del trabajo de parto fue menor en el grupo analgesia peridural. La intensidad reportada de dolor fue menor en el grupo intervención.

\section{Conclusiones}

El presente artículo agrega evidencia a favor del uso de analgesia peridural, durante el trabajo de parto incipiente, en la reducción del dolor (y quizá duración del mismo), sin agregar comorbilidad ni condicionar la vía final de parto.

Tabla: Resultados principales

\begin{tabular}{|c|c|c|c|c|}
\hline Punto final & $\begin{array}{l}\text { Grupo intervención } \\
\text { N:366 (Analgesia } \\
\text { Peridural temprana) }\end{array}$ & $\begin{array}{c}\text { Grupo control } \\
\text { N: 362(Analgesia } \\
\text { Parenteral temprana) }\end{array}$ & $\begin{array}{l}\text { Diferencia } \\
\text { (IC) }\end{array}$ & $\mathbf{p}$ \\
\hline Tasa Cesárea & $17,8 \%$ & $20,7 \%$ & $-2, \%(-9$ a 3$)$ & 0.31 \\
\hline Apgar $<7$ a $5 \mathrm{~min}$ & 1,4 & 2,5 & $-1,1(-3,4$ a 1,1$)$ & 0.28 \\
\hline
\end{tabular}

\section{Comentario}

El trabajo de parto constituye una de las instancias de mayor dolor durante la vida de una mujer (sino la más dolorosa). Desde los tiempos del "parirás con dolor", hasta las actuales y las modernas técnicas de analgesia disponibles, el paradigma de atención del trabajo de parto y manejo del dolor ha ido cambiando. Aun así, existe controversia con respecto al tipo de analgesia a utilizarse e inclusive en algunos ámbitos todavía se discute si hay que usarla. El Colegio Americano de Ginecología y Obstetricia (ACOG) recomienda, "cuando sea posible, retrasar la administración de anestesia peridural en mujeres nulíparas hasta lograr una dilatación cervical de $4-5 \mathrm{~cm}$, utilizando otras formas de analgesia hasta entonces".'

A nivel local, una guía inter-sociedades creada para el Ministerio de Salud de la Nación Argentina recomienda priorizar técnicas no farmacológicas (posicionales, deambulación, acompañamiento familiar, etc.); evitar el uso de drogas analgésicas por vía sistémica (ej. meperidina); y la no utilización rutinaria de la analgesia peridural, para lo cual debe haber al menos $4-5 \mathrm{~cm}$ de dilatación cervical ${ }^{2}$. Estas recomendaciones tratan de minimizar los supuestos riesgos de la anestesia peridural (mayor tasa de partos por cesárea, mayores índices de parto vaginal instrumental, mayor utilización de ocitocina, mayor posibilidad de episodios febriles intraparto y mayor duración del mismo).

\section{Conclusiones del comentador}

Así expuestos, los resultados del presente artículo están en concordancia con otros trabajos similares ${ }^{3,4}$, que sustentan el uso precoz de analgesia raquídea y desestiman complicaciones tales como el mayor índice de cesáreas. Y si bien pueden esgrimirse desde el punto de vista técnico algunos puntos de discusión a favor o en contra de la implementación de esta práctica (que seguramente se disipará con el aporte de nuevos trabajos), quizá sea más importante considerar las limitaciones que impone nuestra realidad social al acceso a la analgesia peridural, sobre todo en el ámbito del sistema público, aún en la segunda mitad del trabajo de parto en la cual la indicación de la misma no presenta controversias.

Villalón Gabriel [ Unidad de Medicina Familiar y Preventiva. Hospital Italiano de Buenos Aires. ]

Villalón,G.La analgesia peridural iniciada en fases tempranas del trabajo de parto no aumenta el riesgo de cesárea. Evid. actual. práct. ambul. 2005;8:105. Comentado de Wong CA, Scavone BM, Peaceman AM. The Risk of Cesarean Delivery with Neuraxial Analgesia Given Early versus Late in Labor. NEJM 2005;352:655-65.

\section{Referencias}

1. GoetzI L, ACOG Practice Bulletin no.36: clinical management guidelines for obstetrician-gynecologist: obstetric analgesia and anesthesia. Obstet Gynecol $2002 ; 100: 177-91$.

2. Uranga A, Urman J, Lomuto C. Guía para la atención del parto normal en maternidades centradas en la familia. Dirección Nacional de Salud Materno Infantil. Ministerio de Salud, Argentina 2004.

3. Grant G. Management of pain during labor and delivery. En: Uptodate, Vol 12 № 3.

4. Grant G. Prevention and treatment of adverse effects of neuraxial anesthesia. En: Uptodate, Vol 12 № 3. 